\title{
MONOTONICITY OF SEQUENCES INVOLVING CONVEX FUNCTION AND SEQUENCE
}

\author{
F. QI AND B.-N. GUO
}

Abstract. Let $f$ be an increasing convex (concave, respectively) function defined on $[0,1]$ and $\left\{a_{i}\right\}_{i \in \mathbb{N}}$ be an increasing positive sequence such that $\left\{i\left(\frac{a_{i}}{a_{i+1}}-1\right)\right\}_{i \in \mathbb{N}}$ decreases $\left(\left\{i\left(\frac{a_{i+1}}{a_{i}}-\right.\right.\right.$ 1) $\}_{i \in \mathbb{N}}$ increases, respectively), then the sequence $\left\{\frac{1}{n} \sum_{i=1}^{n} f\left(\frac{a_{i}}{a_{n}}\right)\right\}_{n \in \mathbb{N}}$ is decreasing. Let $f$ be an increasing convex (concave, respectively) positive function defined on $[0,1]$ and $\varphi$ be an increasing convex positive function defined on $[0, \infty)$ such that $\varphi(0)=0$ and the sequence $\left\{\varphi(i)\left[\frac{\varphi(i)}{\varphi(i+1)}-1\right]\right\}_{i \in \mathbb{N}}$ decreases, then the sequence $\left\{\frac{1}{\varphi(n)} \sum_{i=1}^{n} f\left(\frac{\varphi(i)}{\varphi(n)}\right)\right\}_{n \in \mathbb{N}}$ is decreasing.

As applications, taking special sequence $\left\{a_{i}\right\}_{i \in \mathbb{N}}$ and special functions $f$ and $\varphi$, many new inequalities between ratios of means are obtained, and the Alzer's inequality, the MincSathre's inequality, and the like, are recovered.

Mathematics subject classification (2000): 26D15, 26A51.

Key words and phrases: inequality, convex function, logarithmically convex sequence, ratio of means, monotonicity.

\section{REFERENCES}

[1] H. Alzer, On an inequality of H. Minc and L. Sathre, J. Math. Anal. Appl., 179, (1993), 396-402.

[2] T. H. Chan, P. GAO AND F. QI, On a generalization of Martins' inequality, Monatsh. Math., 138, 3 (2003), 179-187. RGMIA Res. Rep. Coll., 4, 1 (2001), Art. 12, 93-101. Available online at URL: http://rgmia.vu.edu.au/v4n1.html.

[3] CH.-P. Chen, F. QI, Notes on proofs of Alzer's inequality, Octogon Math. Mag., 11, 1 (2003), 29-33.

[4] Ch.-P. Chen, F. QI, P. CERONE, AND S. S. DRAGOMIR, Monotonicity of sequences involving convex and concave functions, Math. Inequal. Appl., 6, 2 (2003), 229-239. RGMIA Res. Rep. Coll., 5, 1 (2002), Art. 1, 3-13. Available online at URL: http://rgmia.vu. edu . au/v5n1 . html.

[5] N. Elezović, J. PeČArić, On Alzer's inequality, J. Math. Anal. Appl., 223, (1998), 366-369.

[6] B.-N. GuO, F. QI, An algebraic inequality, II, RGMIA Res. Rep. Coll. 4, 1 (2001), Art. 8, 55-61. Available online at URL: http://rgmia.vu.edu.au/v4n1.html.

[7] J.-Ch. Kuang, Chángyòng Bùděngshì (Applied Inequalities), 2nd edition, Hunan Education Press, Changsha City, China, 1993. (Chinese)

[8] J.-Ch. Kuang, Some extensions and refinements of Minc-Sathre inequality, Math. Gaz. 83, (1999), $123-127$.

[9] J. S. MARTINS, Arithmetic and geometric means, an applications to Lorentz sequence spaces, Math Nachr. 139, (1988), 281-288.

[10] H. Minc, L. Sathre, Some inequalities involving $(r !)^{1 / r}$, Proc. Edinburgh Math. Soc. 14, $(1964 / 65)$, $41-46$.

[11] D. S. Mitrinović, J. E. PeČARIĆ AND A. M. FinK, Classical and New Inequalities in Analysis, Kluwer Academic Publishers, Dordrecht/Boston/London, 1993.

[12] N. OzEKI, On some inequalities, J. College Arts Sci. Chiba Univ. 4, 3 (1965), 211-214. (Japanese) 
[13] F. QI, An algebraic inequality, J. Inequal. Pure Appl. Math. 2, (2001), Art. 13. Available online at URL: http://jipam.vu.edu.au/article.php?sid=129. RGMIA Res. Rep. Coll. 2, 1 (1999), Art. 8, 81-83. Available online at URL: http://rgmia.vu .edu.au/v2n1.html.

[14] F. QI, Generalization of H. Alzer's inequality, J. Math. Anal. Appl. 240, (1999), 294-297.

[15] F. QI, Generalizations of Alzer's and Kuang's inequality, Tamkang J. Math. 31, 3 (2000), 223-227. RGMIA Res. Rep. Coll. 2, 6 (1999), Art. 12, 891-895. Available online at URL: http://rgmia.vu.edu.au/v2n6.html.

[16] F. QI, Inequalities and monotonicity of sequences involving $\sqrt[n]{(n+k) ! / k !}$, Soochow J. Math. 29, 4 (2004), 353-361. RGMIA Res. Rep. Coll. 2, 5 (1999), Art. 8, 685-692. Available online at URL: http://rgmia.vu.edu.au/v2n5.html.

[17] F. QI, On a new generalization of Martins' inequality, RGMIA Res. Rep. Coll. 5, 3 (2002), Art. 13, 527-538. Available online at URL: http://rgmia.vu.edu.au/v5n3.html.

[18] F. QI, L. DEBNATH, On a new generalization of Alzer's inequality, Internat. J. Math. Math. Sci., 23, 12 (2000), 815-818.

[19] F. QI, B.-N. GuO, An inequality between ratio of the extended logarithmic means and ratio of the exponential means, Taiwanese J. Math., 7, 2 (2003), 229-237.

[20] F. QI, B.-N. GUO, Monotonicity of sequences involving convex function and sequence, RGMIA Res. Rep. Coll. 3, 2 (2000), Art. 14, 321-329. Available online at URL: http: //rgmia .vu .edu . au /v3n2 .html.

[21] F. QI, Q.-M. LuO, Generalization of H. Minc and J. Sathre's inequality, Tamkang J. Math. 31, 2 (2000), 145-148. RGMIA Res. Rep. Coll. 2, 6 (1999), Art. 14, 909-912. Available online at URL: http://rgmia.vu.edu.au/v2n6.html.

[22] J. SÁNDOR, On an inequality of Alzer, J. Math. Anal. Appl. 192, (1995), 1034-1035.

[23] J. SÁNDOR, Comments on an inequality for the sum of powers of positive numbers, RGMIA Res. Rep. Coll. 2, 2 (1999), 259-261. Available online at URL: http://rgmia.vu. edu .au/v2n2.html.

[24] J. S. UME, An elementary proof of H. Alzer's inequality, Math. Japon. 44, 3 (1996), 521-522.

[25] Z. Xu, D. Xu, A general form of Alzer's inequality, Comput. Math. Appl. 44, (2002), 365-373. 